\title{
ATROPHIC SCLEROSIS OF THE CEREBRAL CORTEX ASSOCIATED WITH BIRTH INJURY
}

\author{
BY
}

R. M. NORMAN, M.D.

\author{
(From the Burden Mental Research Department, Stoke Park Colony, Bristol)
}

\begin{abstract}
Although the cerebral lesions of birth injury in the new born have been well studied, there is still considerable uncertainty as to the nature of their late neuropathological sequelae. This paper is concerned with one aspect only of this problem, namely, the relationship of birth injury to a particular histological type of cortical atrophic sclerosis (ulegyria) which may produce the clinical picture of cerebral diplegia or of cortical blindness. This form of gyral atrophy is uncommon, having been encountered only four times in $\mathbf{3 0 0}$ autopsies at Stoke Park.
\end{abstract}

Ford (1927) has given a good acount of the controversies as to whether atrophic sclerosis follows vascular accidents of birth or prenatal degeneration of neurones. McNutt's (1885) influential paper had stressed the importance of meningeal haemorrhage as a precursor of gyral atrophy, an explanation accepted by Gowers (1893) and Osler (1889). Collier's (1924) address to the Royal Society of Medicine did much to discredit the theory of meningeal haemorrhage, but his conclusion that atrophic sclerosis resulted from a primary degeneration of the nerve cells in foetal life did not take into account the other possible consequences of birth injury and failed to carry general conviction (Sachs, 1926; Wilson, 1940).

The investigations of Schwartz (1924, 1927; Schwartz and Fink, 1926) on the brains of infants dying at, or shortly after, birth put the pathology of birth trauma on a more solid foundation and his work has opened up new fields of research to the student of mental deficiency. Schwartz showed the hitherto unsuspected frequency of multiple intracerebral haemorrhages and softenings which occurred especially in the periventricular drainage area of the tributaries of the veins of Galen, and he also described diffuse lesions of the cortex which apparently represented early stages of atrophic sclerosis. In a series of older individuals he was able to trace the evolution of these recent lesions to their terminal states of porencephaly or sclerosis. He illustrated examples of gyral shrinkage and distortion, often associated with an apparent multiplicity of small convolutions and affecting in particular the parietal and occipital lobes.

This condition, named by Schwartz 'sclerotic microgyria,' appears to be identical macroscopically with that found in the cases described in this paper.
The controversial problem is whether or not such changes are pathognomonic of birth injury, for there is no doubt that there are several etiologically distinct forms of ulegyria: prenatal, natal and postnatal varieties certainly exist. Since information concerning the early histories of cases coming to post-mortem examination in later life is often vague or equivocal, there is urgent need for pathological criteria by which the cortical sclerosis of birth injury may be recognized. This task of classification is complicated by the knowledge that functional circulatory disturbance probably plays a part in many forms of infantile encephalopathy, so that even if a lesion is shown to be of vascular origin it does not necessarily follow that trauma at birth is the responsible precipitating factor.

As a result of these considerations, it has been thought profitable to record three examples of gyral atrophy which have in common certain histological features indicative of a vascular pathogenesis, and to discuss the significance of birth injury in their etiology. It is by the collection of individual cases of this sort that a more precise classification of the causes of severe mental defect may eventually be made.

\section{Report of cases}

Case 1. P. S., a male, was admitted to Stoke Park Colony when 9 years old and died aged 16 years 5 months.

History prior to admission. The following information was given by the mother. The child was first born and illegitimate. He weighed $4 \frac{1}{2} \mathrm{lb}$. at birth, labour pains lasting only two hours. For nearly nine months he "did nothing but scream and cry.' He suffered from frequent severe epileptic fits which twice necessitated the administration of chloroform. In the fourth year of life he "seemed to improve a great deal,' but was still unable to attempt walking. There was no febrile illnesses during infancy. The father, ' a terrible drunkard,' deserted the mother and no further information concerning him is available.

Examination after admission. The patient was classed as an imbecile, having a mental age of 2 years 7 months as estimated by the Merrill-Palmer Scale in which he was undoubtedly handicapped by 
physical disability, being able to use only his left hand in the performance tests. Speech was confined to the repetition of a few poorly articulated words. Neurological examination revealed an asymmetrical cortico-striate diplegia. The pupils reacted normally to light. Ocular co-ordination was poor and an alternating divergent squint was present. On smiling, the left side of the face moved considerably more than the right. Power of movement was greatly reduced in the right arm, the tendon reflexes of which were diminished by a plastic type of rigidity.
Athetoid movements of the fingers were noted. In the left arm, movements and co-ordination were comparatively good. Spastic paresis of the legs was present with contractures of hamstrings and tendo Achillis of both sides. The patient could raise his legs but could not walk, stand, or sit up, without help. The knee jerks were equally brisk and obtainable from the whole extent of the subcutaneous surface of each tibia. The left ankle jerk set off ankle clonus. An extensor plantar response and Oppenheim's sign were noted on the left side. The
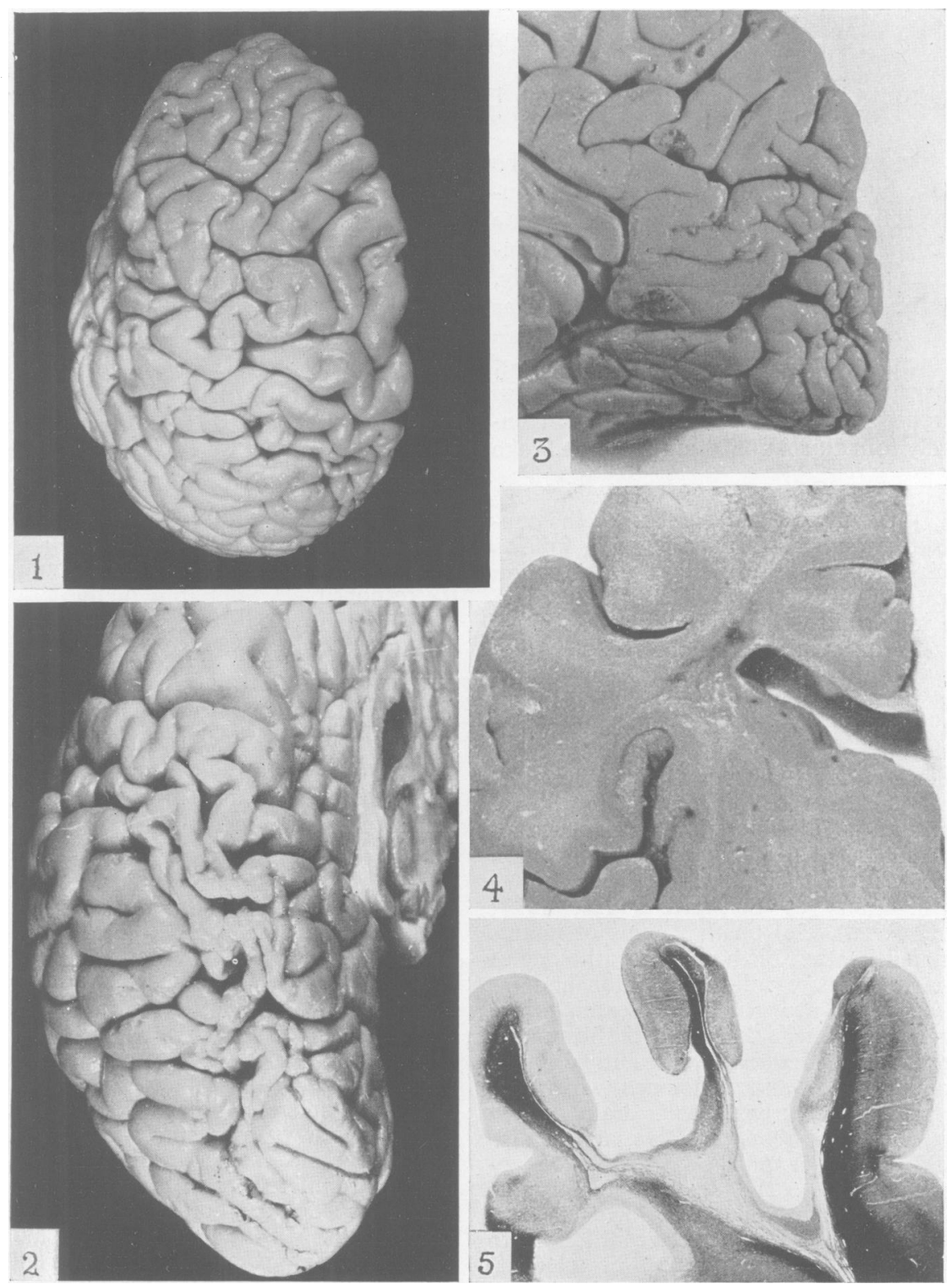

Fig. 1.-Case 1: Left hemisphere showing abnormal gyral pattern of the frontal lobe.

Fig. 2.-Case 1: Ulegyria of the left parieto-occipital area.

Fig. 3.-Case 1: Ulegyria of the right calcarine cortex.

FiG. 4.-Case 1: Coronal section of the left frontal lobe showing area of periventricular softening and dilatation of the lateral ventricle.

FIG. 5.-Case 1: Ulegyria of the left parietal cortex. Note the narrow streaks of demyelinization affecting the central cores of the two gyri on the left and the hemiatrophy of the gyrus on the right. Anderson's modified Kultschitsky-
Pal stain $\times 5 \cdot 5$. 
Wassermann reaction of the C.S.F. was negative and the fluid was also normal in other respects.

Prior to his death, which resulted from the perforation of an oesophageal ulcer, his mental and physical condition showed no obvious change, and there is no record of his having had an epileptic fit while in the Colony.

Pathological examination of the central nervous system.

MACROSCOPIC EXAMINATION. The brain was small, weighing with cerebellum and brain stem $1126 \mathrm{gm}$. Dura and leptomeninges were normal. There was conspicuous shrinkage and distortion of the gyri of the left superior parietal lobule at the margin of the great longitudinal fissure, involving the superior end of the post central gyrus and reaching posteriorly to within $2 \mathrm{~cm}$. of the occipital pole (fig. 2). A similar condition was present on the medial aspect of the right hemisphere on both sides of the parieto-occipital fissure and along the beginning of the upper lip of the posterior calcarine fissure (fig. 3). On sectioning the brain in these areas it was seen that the deeper parts of the walls of these small gyri were more shrunken than their crowns. The point of transition between their bulbous crowns and elongated sclerotic stalks was often abrupt and sometimes a symmetrically placed indentation occurred on either side of the gyrus, producing, as it were, a neck beneath the expanded terminal portion. The pattern of the left frontal lobe was abnormal, there being little indication of a superior or inferior frontal sulcus (fig. 1). The right frontal lobe appeared more normal, though here also the superior frontal sulcus was short and ran obliquely into the great longitudinal fissure. On sectioning the brain in the coronal plane, symmetrically placed greyish areas of old softening were seen in the central white matter adjoining the anterior horns of the lateral ventricles, which were moderately dilated. This periventricular lesion was more marked on the left side (fig. 4). The body of the corpus callosum was thinner than usual for a brain of this size.

MicROSCOPIC EXAMINATION. Blocks of tissue were taken from representative areas of the cerebral cortex of both hemispheres, from different parts of the basal ganglia, brain stem and cerebellum. Sections were stained by standard methods for nerve cells, axis cylinders, neuroglia and lipoid.

CEREBRAL CORTEX. Examination of the left parieto-occipital and right occipital regions showed that the atrophied parts of the cortex had in common certain distinguishing features. The shrunken, constricted basal portions of such gyri showed intense neuroglial sclerosis with conspicuous sheaves of piloid glia separating an outer compact cortical layer from a deeper cystic part (fig. 8). In such areas nerve cells and myelinated fibres were usually absent, though in less severely affected gyri a few clusters of pyramidal cells remained, being sometimes arranged in two thin layers between which a streak of neuroglial scar passed upwards towards the crown of the convolution. Similar thin streaks of atrophy were seen in the white matter of the central cores (fig. 5). A small, sharply demarcated focus of total demyelinization * was usually present below the base * In order to avoid a cumbersome terminology, this term has been used to denote an absence of myelin. If the lesion occurred at, o myelinated. of the shrunken gyrus. Here and there were scavenger cells containing lipoid. Holzer staining showed that dense neuroglial proliferation corresponded to this subcortical area of demyelinization, while a moderate gliosis affected the neighbouring white matter to a variable extent. The crowns of the atrophied gyri were often split by superficial indentations into a series of smaller convolutions, thus giving an abnormal appearance of complexity to the surface of the brain (fig. 6). Neuroglial sclerosis ceased abruptly at the margin of these nerve cell bearing parts of the cortex where, in myelin preparations, an abnormal density of darkly staining fibres was often seen (figs. 6 and 7).

The transition between these ulegyric parts and the macroscopically normal gyri was usually abrupt. Sometimes one half of a large convolution was totally atrophied, the remainder being normal, apart, perhaps, from a small hypermyelinated scar (fig. 5). It was found on microscopic examination that the sharp constrictions often seen in the walls of the less affected gyri corresponded to small, discrete neuroglial scars which were associated with hypermyelinized axis cylinders and formed short, laminar defects in the cortex, usually in its third layer. Another variation of this theme is seen in fig. 6 and 7 . Here narrow neuroglial scars emerge from the area of subcortical sclerosis and curve upwards for a short distance into the wall of a neighbouring gyrus.

The parts of the cerebral cortex remote from these ulegyric areas showed little evidence of pathological change. In the left cornu Ammonis, however, severe neuroglial sclerosis was found throughout the major part of its extent and there was a typical pyramidal cell defect in the Sommer sector. The macroscopically normal paracentral convolution on the right side proved on section to contain a narrow band of laminar atrophy affecting the middle of the third layer accompanied by coarse, darkly staining myelinated fibres running parallel to its course. A mild, diffuse outfall of nerve cells affecting the third cortical layer and unaccompanied by glial proliferation was occasionally seen in sections taken from the frontal lobes of both sides. A few gitter cells containing lipoid were often seen around the small blood vessels of the central cores of white matter of gyri which were apparently normal in other respects.

CENTRAL WHITE MATTER. Gross demyelinization and neuroglial scarring were present in the areas of softening adjoining the lateral ventricles in the frontal lobes. In the loose mesh-works of this gliosis there were scattered scavenger cells containing lipoid. Thick sections showed a few poorly-staining and beaded myelinated fibres traversing the softened areas. The ' $U$ ' fibres of the cortex adjacent to this area of softening were intact.

BASAL GANGLIA. The left thalamus showed marked nerve cell loss in the lateral nucleus associated with dense gliosis. Many of the remaining nerve cells were calcified. Myelinated fibres were not obviously reduced in this area, but no état marbré was seen. The external geniculate body was intact, as were putamen, globus pallidus and caudate nucleus of the left side.

The right thalamus showed a small area of complete demyelinization and neuroglial sclerosis in the dorsal part of the lateral nucleus (fig. 10). A few calcified nerve cells were present near this focus. The external geniculate showed degeneration (retrograde) and gliosis in its deeper laminae. In the 

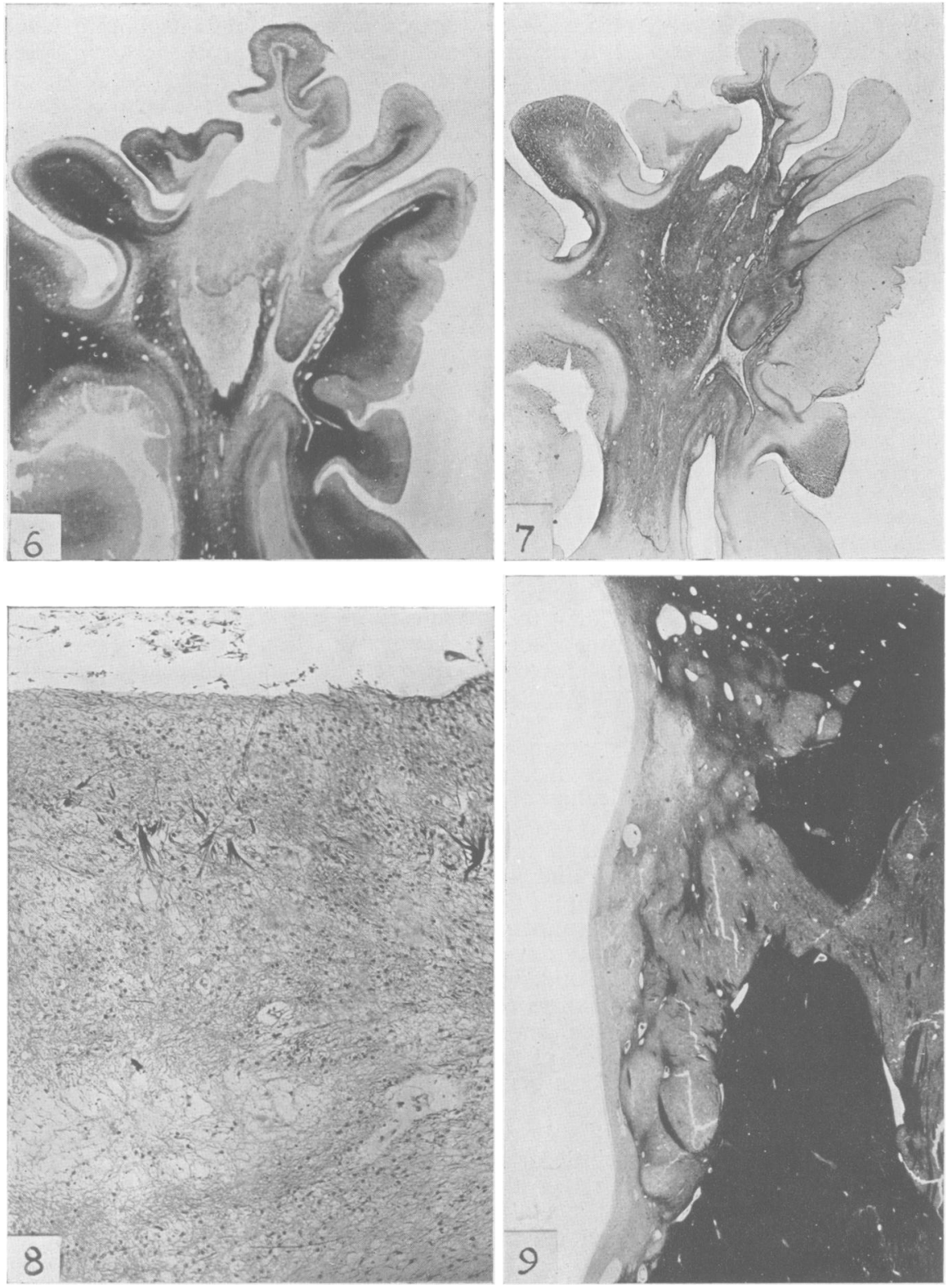

Fig. 6.-Case 1: Sclerotic microgyria of the right occipital lobe. Kultschitsky-Pal $\times 3$.

FIG. 7.-Case 1: Adjoining section to that shown in fig. 6 . Holzer stain $\times 3$.

FIG. 8.-Case 1: Gliosis in the wall of an atrophied gyrus. Mallory's phosphotungstic acid hæmatoxylin $\times 80$.

Fig. 9.-Case 1: Right caudate nucleus. Coronal section showing état marbré. Kultschitsky-Pal $\times 5$.

right caudate nucleus a small focus of necrosis, similar to that seen in the thalamus, was found at the junction of head and body. Radiating from this area there was a well-marked état marbré (fig. 9). The putamen and globus pallidus were intact.

BRAIN STEM. The left pyramidal tract was smaller and slightly less well myelinated than the right. This abnormality was seen in sections from the pons, medulla, and cervical cord, but no demonstrable gliosis was present.

Case 2. G. H., a male, was admitted to the Colony when 7 years 10 months of age and died one month later.
History prior to admission. The patient was the firstborn child of highly intelligent parents whose family history was free from neuropathic taint. Another sib, four years younger, is normal. Both parents were interviewed and gave me the following information:-

The mother's age at the birth of her son was 36 years. During pregnancy her health was good apart from troublesome vomiting for the first four months. The baby was full term. Labour pains began at 3 a.m. and at 11.30 a.m. the family doctor delivered the child by forceps.

The child was apathetic for the first two days. His condition then deteriorated, he became weak 
and unable to take his feeds and was admitted to the Birmingham Children's Hospital under Prof. L. G. Parsons, to whom I am indebted for the following notes:- ' On admission he was a normally developed baby but slightly jaundiced; there was no bulging of the fontanelle. On the day after admission he had some slight twitching of the right eye and right leg, but this did not recur. The child was in hospital for six days, during which time he put on $3 \mathrm{oz}$. in weight.' During his stay in hospital he was fed by his mother's milk and when he came home took the breast fairly well. There was no obvious paralysis, but he had a fit when six weeks old. Frequent fits were noted from the age of 4 months and at 7 months they were of daily occurrence. He paid no attention to moving objects, nor could he sit up or even raise his head for very long.

Examination after admission. A thin, apathetic child with slight pigeon breast. Height $3 \mathrm{ft}$. 6 in.; weight 2 st. 1 lb. A neurological examination showed a generalized excessive muscle tone, all tendon reflexes being markedly exaggerated. The abdominal reflexes were absent and both plantar responses were extensor. There was some contracture of the hamstrings. He was incapable of purposive movement and exhibited coarse, irregular jerky movements of choreic type, present at rest and considerably exacerbated on cutaneous stimulation. He could not hold up his head or sit up and was doubly incontinent. The skull was asymmetrical in that the right occipito-parietal region was relatively flattened.

The blood Wassermann reaction was negative.

On admission he had to be bottle fed but later became capable of swallowing fluids by spoon. Several major and a few minor fits occurred daily until controlled by luminal. Death resulted from bronchopneumonia.

\section{Pathology of the central nervous system.}

MACROSCOPIC EXAMINATION OF THE BRAIN. Weight $1105 \mathrm{gm}$. (left hemisphere $460 \mathrm{gm}$.; right hemisphere $510 \mathrm{gm}$.; cerebellum and brainstem $135 \mathrm{gm}$.). The leptomeninges showed slight thickening and oedema. The left postcentral gyrus was narrow and shrunken, its crown showing two shallow, irregular grooves running parallel with the long axis of the convolution. The gyri of the left frontal lobe were somewhat narrower and more tortuous than those of the rignt side. On coronal section, shrinkage of the deeper parts of the gyri of the left frontal lobe was apparent, the posterior portions of the second and third frontal convolutions being affected in this manner, the gyral crowns, however, being somewhat swollen and friable. There was slight dilatation of the left frontal horn of the lateral ventricle.

MicrosCopic EXAMINATION. CEREBRAL CORTEX. The leptomeninges showed mild fibrous thickening but were not adherent to the cortex. The anterior wall and crown of the left postcentral gyrus showed dense myelinated fibres arranged in a narrow band occupying the middle of the grey matter (fig. 11). Holzer staining demonstrated thin glial scars corresponding to the position of this abnormal myelinization. In this part of the grey matter nerve cells were absent and a diffuse loss of neurones was also seen in the adjoining cortex. In the proximity of arterioles in the sclerosed areas a few scavenger cells filled with lipoid were commonly present. The frontal gyri of the left side also showed several myelinated scars of the same type, more particularly in their walls (fig. 12 and 13). It was observed that in the parts of the grey matter subjacent to these linear defects the normal radial fibres were absent, their place being taken by numerous irregularly arranged myelinated axis cylinders. The cortex above the scar, however, was almost devoid of myelin except for a narrow layer of tangential fibres near the surface of the gyrus.

In general, the nerve cells of the cortex stained very poorly with cresyl violet or toluidine blue, the cell bodies being scarcely visible. The Betz cells stained diffusely and did not appear to be numerically deficient. Both hippocampi and visual areas were intact. Arterioles and often capillaries were congested and signs of terminal oedema of the brain were evident in the form of dilated perivascular and pericellular spaces. A diffuse loss of cells in the third layer, unaccompanied by gliosis, was commonly seen in the frontal and parietal lobes.

OTHER PARTS OF THE BRAIN. Sections of the left thalamus made in the coronal plane at the level of the external geniculate body showed conspicuous atrophy of nerve cells with accompanying gliosis in the region of the nucleus ventralis posterolateralis. In the medulla both pyramidal tracts were well myelinated, the left, however, being the smaller. Sections from cervical, thoracic and lumbar levels of the spinal cord revealed no abnormality with regard to myelin, glia or nerve cells.

Case 3. H. M., a male, was admitted to the Colony when 15 years old and died aged 17 years 5 months.

History prior to admission. The patient was the sixth child of a family of seven. The mother's age was 38 years at the time of his birth. The other six sibs were mentally and physically normal according to the mother (1943) who has supplied the following information. The infant was full term. The mother could recollect no details about the birth, which she regarded as normal. The infant took the breast well and no anxiety was experienced until the fourth day, when a convulsion occurred and it was noticed that the baby was jaundiced. The mother cannot remember how long the jaundice persisted, but the child's health was apparently unaffected. Fits recurred frequently during infancy-'Every day, but sometimes he would have them more than I can remember.'

He began to walk at the age of three years.

Examination after admission. The patient was classed as an imbecile verging on idiocy, but on account of grossly impaired vision an accurate estimate of his mental abilities was impossible: Speech was limited and articulation defective. He could not feed or dress himself. Spontaneous activity was largely limited to rocking himself forwards and backwards on his chair. Physically he was undersized and small headed. A neurological examination at this time gave the following information. Pupils reacted to light but no response was elicited to accommodation as he was unable to fixate an object. There was an alternating divergent strabismus, and an error of refraction of about +5 in both eyes. The fundi were difficult to examine owing to constant rolling of the eyes under the lids but the discs were seen to be pale, though not definitely atrophic (Mr. A. E. Iles). In the upper 

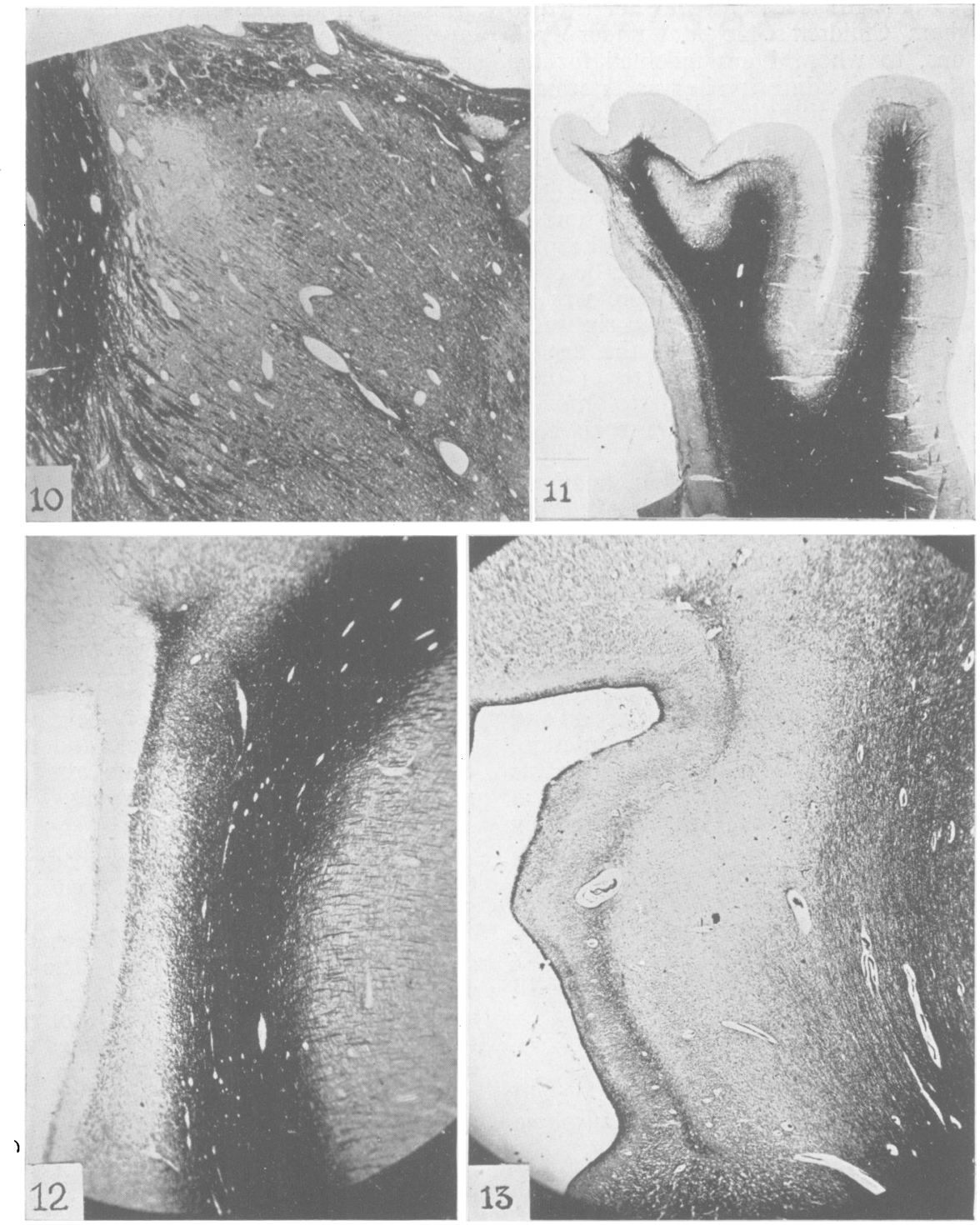

Fig. 10.-Case 1: Right thalamus showing small focus of necrosis. Kultschitsky-Pal $\times 6$.

Fig. 11.-Case 2: Left post-central gyrus (on left) showing laminar hypermyelinization. Kultschitsky-Pal $\times 3$.

Fig. 12.-Case 2: Left frontal cortex showing laminar hypermyelinization in middle zone of grey matter in gyral wall. Kultschitsky-Pal $\times 13$.

Fig. 13.-Case 2: As in fig. 12 (adjacent part of block) showing glial scar. Mallory's phosphotungstic acid haematoxylin $\times 13$.

limbs the muscle and tendon reflexes were brisk and equal. In the legs the knee jerks were equal and exaggerated, being obtained from the whole length of the leg. Ankle jerks were also equal and very brisk. The abdominal and cremasteric reflexes were present on both sides. An extensor plantar reflex was obtained on the left side.

During his life in the Colony his neurological condition remained stationary and he suffered from major epileptic fits at irregular intervals. Death resulted from phthisis.

\section{Pathology of Central Nervous System.}

MACROSCOPIC EXAMINATION OF THE BRAIN. The brain was small for a boy of 17 years, weighing only $1061 \mathrm{gm}$. with cerebellum and brainstem. The

region of the left posterior calcarine fissure was occupied by a group of minute convolutions (fig. 14). A similar appearance was presented by the lower lip of the calcarine fissure of the opposite side, and a few small formations of the same sort were just visible on the surface of the right hemisphere emerging from the upper part of the post-central sulcus. Sclerotic atrophy was evident in the depths of some of the sulci in the left precuneal region. The general convolutional pattern of the brain was not otherwise abnormal, except perhaps in the right temporal lobe where the superior temporal sulcus was unusually short.*

* A photograph of this hemisphere appears in 'A cerebral Atlas.' Berry, R. J. A., Oxford University Press, 1938. Fig. 223. 

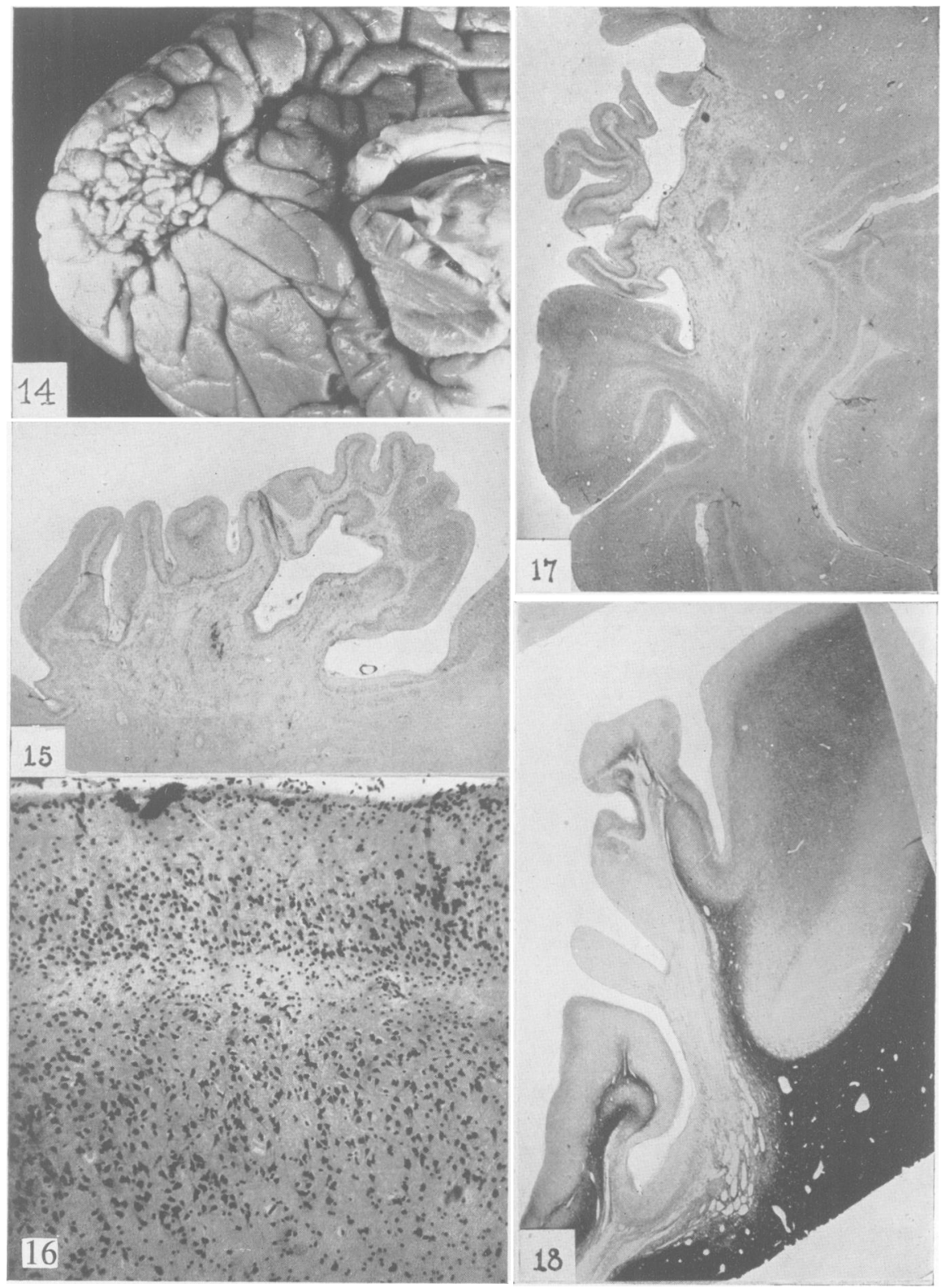

Fig. 14.-Case 3: Left calcarine area showing microgyria.

FIG. 15.-Case 3: Microgyria from left occipital lobe. Cresyl violet $\times 4 \cdot 4$.

Fig. 16.-Case 3: Laminar defect in cortex of left precuneal region. Cresyl violet $\times 60$. Note the irregular disposition of the nerve cells in the deeper layers.

Fig. 17.-Case 3: Right occipital lobe. Coronal section showing microgyria filling in the defect formed by the atrophy of the calcarine cortex. Cresyl violet $\times 3.7$.

FIG. 18.-Case 3: Microgyria arising from the posterior wall of the right post-central gyrus. Kultschitsky-Pal $\times 4$.

Microscopic eXAmination. Sections prepared from the microgyric areas of both occipital poles and the upper right postcentral area revealed a type of structure already encountered in case 1 , with this qualification, that whereas in the first case the crowns of the sclerosed gyri usually showed only shallow superficial indentations, in the present instance the process of splitting into small subconvolutions had often proceeded further and had resulted in the creation of numerous, discrete microgyric formations. In nerve cell preparations these were seen to consist of thin loops of pyramidal cells, sometimes having a wavy or festoon-like outline (fig. 15). The common stem from which these small gyri sprang was invariably heavily sclerosed, the edge of the neuroglial proliferation ceasing abruptly at the neurone-bearing crowns, where dense whorls of hypermyelinated fibres were often present. The striate areas of both sides were extensively atrophied, each posterior calcarine fissure being 

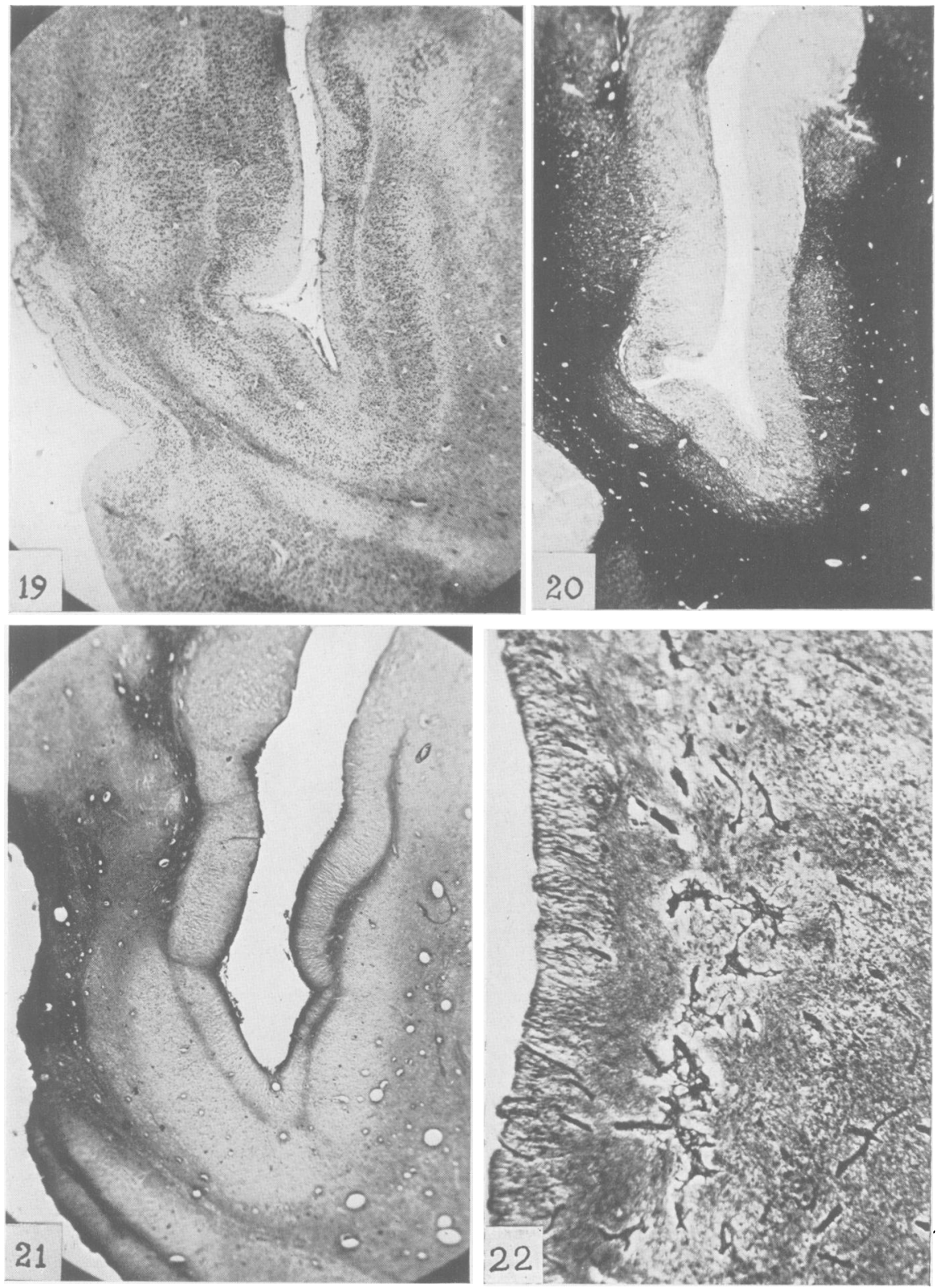

Fig. 19.-Case 3: Laminar defect in the depths of a gyrus in the left precuneal region. Cresyl violet $\times 12 \cdot 8$.

Fig. 20.-Case 3: Adjoining section to that of fig. 19. Kultschitsky-Pal $\times 12 \cdot 8$.

FIG. 21.-Case 3: As in fig. 20. Holzer stain $\times 12 \cdot 8$.

FIG. 22.-Case 3: Sclerotic blood vessels in a laminar defect of the cortex. Hortega's silver carbonate method for astrocytes $\times 60$.

represented by a depression filled with the microgyric convolutions already described, which appeared to have grown down from the region of the parietooccipital fissure and to have filled up the space left by the destruction of the calcarine visual area (fig. 17). The anterior calcarine fissures were macroscopically normal but the gyri on either side occasionally showed short linear hypermyelinated scars in their walls. Laminar atrophy of the cortex was a conspicuous feature of the neighbouring occipital gyri (fig. 17) and was also present in the depths of the gyri of the left precuneal area where ' $U$ '-shaped cortical defects could be seen by the naked eye in the depths of the sulci. The nerve cell, myelin and glial, abnormalities of such regions are shown in fig. 16, 19, 20 and 21. It will be seen that the cortex is divided into two layers by a dense neuroglial scar, the inferior limit of which is occupied (in myelin preparations) by a line of darkly myelinated fibres. In the deeper layers of the cortex the normal radial bundles are replaced by a close network of fibres which occasionally show typical plaques fibromyéliniques. The transition between this laminar defect and the normal cortex is abrupt and occurs at the fourth, or granular layer. The blood vessels in the linear streaks of atrophy showed 
adventitial thickening (fig. 22). As in the other cases, a few compound granular corpuscles were sometimes seen near the walls of blood vessels.

The microgyria of the right parietal area originated from the posterior wall of the postcentral sulcus and showed the familiar combination of sclerosis, linear atrophy and hypermyelinization (fig. 18). Nerve cells were diminished in the third layer of the neighbouring pre- and post-central gyri. Elsewhere in the cortex, basal ganglia, cerebellum and brain stem no evidence of a destructive process was discovered. Both external geniculate bodies, however, showed extensive retrograde degeneration.

\section{Comment}

In attempting to assess the probability of birth injury having caused the atrophic sclerosis of the present cases, reliance has necessarily to be placed upon indirect evidence. The lesions were of long standing, and although small groups of microglial phagocytes clearly indicated a destructive process, all evidence of haemorrhage, if such had indeed occurred, had long since vanished. The clinical histories did not support the theory of a post-natal pathogenesis, no febrile illness in infancy likely to produce cerebral atrophy having been observed. The family histories gave no hint of a genetic cause for the condition, nor, as will be seen later, were the pathological findings appropriate to an heredodegenerative process. Evidence of a more positive kind in favour of birth injury may be looked for in the birth histories, in the histological character of the ulegyria and in the distribution of the cerebral lesions, for it is well known that birth trauma has a predilection for certain parts of the brain. These points will now be considered in reference to the present cases.

The clinical histories are suggestive of trauma in the first two cases. The rapid birth of a first born infant weighing only $4 \frac{1}{2} \mathrm{lb}$., as in case 1 , is an event fraught with dangerous possibilities. Some degree of prematurity is likely; a condition which predisposes to trauma because of the thinness of skull and weakness of cerebral veins (Ylppö, 1924). Probably the relative insensibility of the respiratory centres to carbon dioxide known to exist in premature infants (Levine and Gordon, 1942) is an added factor of importance. In the second case, that of a first-born male infant, in this instance full term and delivered by forceps, the general condition deteriorated two days after birth, with apathy and refusal to take feeds, as has repeatedly been recorded in birth-injured children (Rydberg, 1932). The slight focal epilepsy displayed in the early days is also indicative of localized brain injury. In the third case there is no history of abnormal labour and the onset of convulsions on the fourth day, though consistent with birth injury, might perhaps with equal justification be ascribed to a prenatal cause.

The histological character of the ulegyria is more in keeping with a vascular than a degenerative pathogenesis. The lesions affected small areas of the cortex, were relatively sharply circumscribed, and were grouped around foci of special intensity which involved the deeper parts of the gyri and adjoining subcortical white matter. This special vulnerability of the gyral wall to anoxaemia has been noted by Doring (1936) in deaths from drowning. No less characteristic of a vascular process was the presence in all three cases of narrow streaks of atrophy usually affecting the middle zone of the cortex and ending abruptly in the granular or fourth layer of neighbouring gyri. Arteriolar lesions of similar distribution are common in cerebral vascular disease of adult life (Neubürger, 1930). Alajouanine and Hornet (1936) have recorded an instructive example of extensive laminar atrophy with a small focus of cortico-subcortical softening in an elderly arteriosclerotic patient. There is a close resemblance between such a picture and the occipital lesions present in case 3. The narrow, elongated hypermyelinized scars, so frequently seen in the present cases, are also a feature of the uncommon form of gyral sclerosis known as 'the granular atrophy of Spatz' (Pentschew, 1933). This condition is characterized by a shrinkage and puckering of the crowns of convolutions and appears to be the consequence of cerebral anoxaemia induced by a variety of predisposing vascular diseases and also by carbon monoxide poisoning.

While there is thus good reason for believing that the atrophic sclerosis in the present cases is the result of a vascular rather than a primarily degenerative process, the problem still remains as to whether these lesions occurred at birth or during the later stages of foetal development. This decision is made the easier in the first case by the fortunate coincidence of subcortical lesions highly characteristic of birth injury, namely, the bilateral periventricular areas of softening in the central white matter of the frontal lobes. In view of Schwartz's observations, there can be little doubt that this condition is the late sequela of haemorrhage or softening in the drainage area of the terminal veins, a conclusion to which further support is given by the glial scarring and foci of necrosis in the right thalamus * and caudate nucleus, the latter associated "with état marbré. Marbling of corpus striatum and thalamus has been recorded in several cases having a history suggestive of birth injury (Scholz et alia, 1938; Meyer and Cook, 1936), but it is rare to find such marked periventricular softening of the white matter in the same brain. Among the many examples of the latter lesion which have been recorded by Schwartz were some which showed, in addition, diffuse sclerosis and shrinkage of cortical gyri. It is thus highly probable that the ulegyria of the parieto-occipital region found in case 1 of the present series dated from a lesion sustained during birth and not from a hypothetical prenatal abnormality. It might be urged that the unusual convolutional pattern of the left frontal lobe (fig. 1) is indicative of prenatal malformation and that * The atrophy affecting the lateral nuclei of the left thalami of clearly examples of retrograde degeneration following partial destruction of the parietal and calcarine cortices respectively. 
lesions found elsewhere in the cortex are, therefore, likely to be of prenatal origin also. Were this case the first of its kind to be reported this argument might have weight, but, as has been pointed out above, the combination of sclerotic cortical lesions and central softening in the distribution of the terminal veins, the latter so typical of birth injury, has been described in other cases by Schwartz. Moreover, variations from the text-book gyral pattern, though common in the brains of mental defectives, also occur in the presumed normal, and their pathological significance, except in gross instances, is uncertain. Even if this gyral configuration be considered an undoubted malformation, this finding is relevant to the pathogenesis of the ulegyria only in as much as malformation may predispose to birth injury. Whilst the possibility of a double pathology for the mental deficiency of the first patient cannot be denied, birth injury still remains the most probable explanation of the cortical atrophy.

In case 2, no such informative periventricular lesions were present, the essentially laminar atrophy being confined to the grey matter of the cortex. Its distribution was consistent with the clinical history which had suggested a mild birth injury affecting the frontal portion of the left hemisphere. The extent of atrophy seems insufficient to account for the idiocy and symmetrical paresis manifested during life. This discrepancy may be made good by taking into account the conspicuous nerve cell loss noted in areas remote from the grosser lesions; but the relationship of this commonplace pathological finding to birth injury is conjectural. It is possible that the diffuse loss of nerve cells in the third layer of the cortex may have been the result of repeated epileptic 'insults,' themselves conditioned by the original foci of sclerotic atrophy. An example of such an association of epilepsy with progressive cortical damage in early infancy has been recorded by Levin (1936).

In case 3 the bizarre form of the microgyria certainly suggests a prenatal origin, and, in a closely similar case (Norman, 1937), that interpretation was formerly favoured. In view of the findings in case 1, it would seem inadvisable to dismiss birth injury from consideration. The clusters of small gyri arising from a common sclerotic stem, which were the characteristic feature of case 3 , may be regarded simply as a further stage in the evolution of such partially split gyral crowns as were observed in case 1. While it is true that the undulating or festoon-like arrangement of the nerve cells in some of the small gyri is reminiscent of what is sometimes found in microgyria of undoubted prenatal origin (Bielschowsky, 1915), the two conditions are dissimilar in other respects. It is unlikely that this sclerotic microgyria was the result of a destructive process superimposed on a pre-existing malformation. On the contrary, the way in which clusters of small convolutions had filled in the depression formed by the atrophied calcarine cortex strongly suggested that the development of the microgyria post-dated an initial lesion of the hemisphere. There is thus good reason for regarding the microgyria of this case also as a postnatal malformation conditioned by a vascular lesion. Although direct proof of birth injury is lacking, it is difficult to suggest a more probable alternative cause.

No attempt will be made here to review the theoretical explanations as to the cause of intracranial haemorrhage or brain softening in the newborn.

Rydberg (1932) has given a good survey of this aspect of the subject. Two points relevant to this discussion may be mentioned. The first is that Holland's (1923) demonstration of the manner in which the great veins of Galen may become obstructed at their junction with the straight sinus owing to excessive moulding of the head seems to provide an adequate explanation for the periventricular haemorrhages so characteristic of birth injury. Secondly, the great diversity of cerebral lesions which may apparently be associated with birth trauma, ranging from massive porencephaly to a microscopic plaque fibromyélinique, is inexplicable on the basis of simple damage to individual blood vessels. Ricker's (1924) conception of functional circulatory disturbance provides a valuable unifying principle, the application of which to the pathology of birth injury clarifies many formerly obscure problems.

\section{Summary}

Clinical and pathological details have been given of three cases of severe mental defect dating from early infancy. The mental defect was associated with cerebral diplegia in the first two cases and cortical blindness in the third. The brains showed a form of atrophic sclerosis or ulegyria affecting relatively small areas of the cerebral cortex in an asymmetrical manner. The atrophy was most marked in the basal parts of the gyri and usually involved a small part of the subjacent white matter. The less affected gyral crowns often showed partial or complete subdivision into smaller microgyric convolutions, which tended to be hypermyelinated. Clear-cut laminar defects in the middle of the grey matter were typical features of the atrophic gyri and were also found radiating from the atrophied parts of the cortex into otherwise intact neighbouring convolutions. These lesions were associated with thin streaks of neuroglial sclerosis and accompanied by darkly staining myelinated fibres running parallel with their course. The first case, in addition to ulegyria, showed lesions in the periventricular drainage area of the terminal veins in the form of bilateral areas of softening in the central white matter of the frontal lobes and small foci of necrosis in the right thalamus and caudate nucleus, the latter accompanied by état marbré. The similarity of these findings with those previously reported by Schwartz in cases of birth injury, together with histories suggestive of trauma in the first two cases, make it highly probable that this form of gyral atrophy is a late sequela of birth injury. 
Thanks are due to Dr. R. M. Bates, Medical Superintendent of Stoke Park, for permission to publish information derived from the Colony records.

\section{REFERENCES}

Alajouanine, T., and Hornet, T. (1936). Rev. neurol., $65,1532$.

Bielschowsky, M. (1915). J. Psychol. Neurol., I.pz., 22, 1.

Collier, J. S. (1924). Brain, 47, 1.

Doring, G. (1936). Virchows Arch., 296, 666.

Ford, F. R. (1927). Cerebral birth injuries, Lond., Medicine monographs, 2.

Gowers, W. R. (1893). A manual of diseases of the nervous system, Lond., 2nd ed.

Holland, E. (1923). J. Obstet. Gynaec., 29, 549.

Levin, P. M. (1936). Arch. Neurol. Psychiat., 36, 264.

Levine, S. Z., and Gordon, H. H. (1942). Amer. J. Dis. Child., 64, 274.
McNutt, S. J. (1885). Amer. J. med. Sci., 89, 58.

Meyer, A., and Cook, L. C. (1936). J. Neurol. Psychopath., 16, 341.

Neubürger, K. (1930). Bumke's Handbuch der Geisteskrankheiten, Bd. II. Berlin.

Norman, R. M. (1937). J. Neurol. Psychiat., 1, 1.

Osler, W. (1889). Cerebral palsies of children, Lond.

Pentschew, A. (1933). Arch. Psychiat. Nervenkr., 101, 80.

Ricker, G. (1924). Pathologie als Naturwissenschaft, Berlin.

Rydberg, E. (1932). Acta path. microbiol. scand., Supp., 10.

Sachs, B. (1926). Amer. J. med. Sci., 171, 376.

Scholz, W., Wake, J., and Peters, G. (1938). Z. ges. Neurol. Psychiat., 163, 193.

Schwartz, P. (1924). Ibid., 90, 263.

(1927). Ergebn. inn. Med. Kinderheilk., 31, 164. , and Fink, L. (1926). $\quad Z$. Kinderheilk., 40, 427.

Wilson, S. A. K. (1940). Neurology, Lond., 2, 760.

Ylppö, A. (1924). $\quad Z$. Kinderheilk., 38, 32. 